\title{
Mastering the donor eye: a new device for obtaining donor corneal discs
}

\author{
ARTHUR D. MCG. STEELE, ${ }^{1}$ AND DERMOT PIERSE ${ }^{2}$ \\ From ${ }^{1}$ Moorfields Eye Hospital, City Road Branch, London, and the Institute of Ophthalmology, University \\ of London; and 'Croydon Eye Unit, Mayday Road, Thornton Heath, Surrey
}

SUMMARY In recognition of the many inexactitudes involved in cutting donor corneal discs, a new instrument is here described which should help to eliminate many of them, and its mode of use is outlined.

For a long time instruments available to assist the corneal surgeon in his management of donor material have been inadequate. Many devices are awkward to use, or give rise to donor discs of an unreliable or uneven edge profile.

In an attempt to overcome these difficulties we have designed, produced and tested a new instrument which we feel represents an advance.

Because different surgeons use fresh or stored material, and because some prefer to trephine from the epithelial surface while others opt to punch from the endothelium, we have endeavoured to meet all these requirements.

\section{Description}

The instrument (Fig. 1) consists of a solid circular base plate, $18.5 \mathrm{~cm}$ in diameter, $2 \mathrm{~cm}$ thick, and weighing $1 \mathrm{~kg}$. The upper surface has 2 holesone central and one close to the edge-in which the moving parts of the instrument fit and are allowed to rotate. Into the central aperture fits a pin which acts as a spindle for the other components, first an optional antifriction ring, then a shallow knurled cylinder (intraocular pressure adjuster) closed at the base except for a hole to fit over the spindle pin. The pin is then surmounted by a distance piece. Sufficient room is left between this spacer and the inner wall of the adjusting ring for the cylinder of the main body, which bears a sliding central block. This block sits on the spacer and is hollowed out at one end to serve as the eye support. The main body has an outer knurled ring, above and below which are screw threads. The lower and deeper thread meets the thread on the inside of the pressure-adjusting ring, while the upper and shallower thread is for the top clamp to be seated securely. This top clamp is a broad ring with a central toothed aperture.

The peripheral hole on the base plate carries a right-angled supporting arm $9 \cdot 5 \mathrm{~cm}$ high which can

Address for reprints: Mr Arthur D. McG. Steele, FRCS, Moorfields Eye Hospital, City Road, London EC1V 2PD

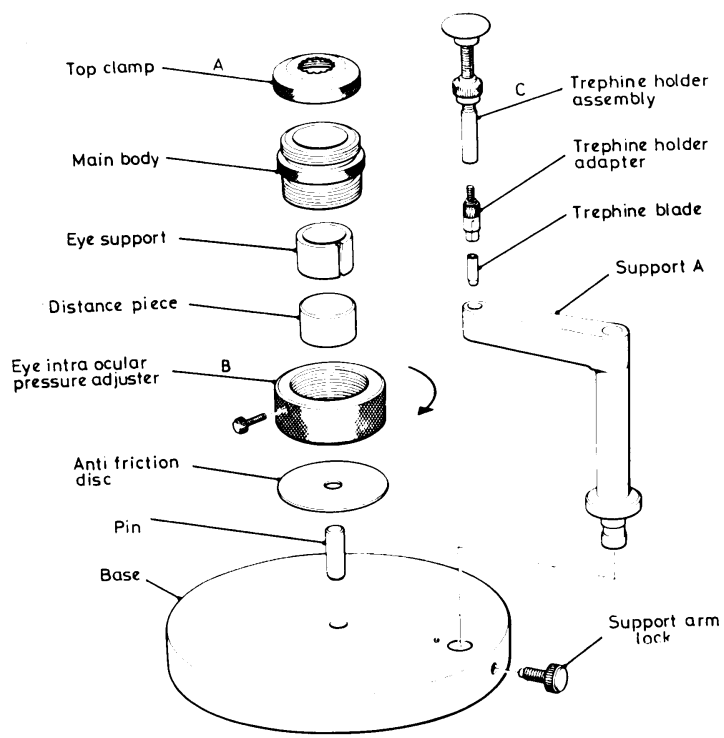

Fig. 1 An 'exploded' diagram of the eye holder

rotate through $90^{\circ}$ from being parallel to the tangent of the base edge to a position precisely centred over the axis of rotation of the central column. This point of the arm has a hole through which the trephine shaft fits. The axis of vertical rotation of the trephine will then exactly correspond with the 
axis of rotation of the central assembly. The supporting arm can be securely locked in this position by a locking nut on the side of the base plate.

The trephine assembly comprises a body which fits exactly the hole in the support arm surmounted by a threaded shaft on which a locking screw, ' $\mathrm{C}$ ', is mounted. The lower end of the body has a threaded recess which carries the various disposable trephine adaptors. At present, adaptors are available for trephines ranging from $6-8 \mathrm{~mm}$ in $0.5-\mathrm{mm}$ steps.

\section{Mode of use}

To cut a penetrating or lamellar disc from a donor eye The central unit is assembled without the top clamp and with the main body engaging very little of the thread on the inner side of the intraocular pressure adjuster.

The donor eye, after cleansing, is then placed in the eye support and the top clamp screwed home over it. Provided the main body has not engaged more than 1 or 2 threads of the adjusting ring, the top clamp will still be $1-3 \mathrm{~mm}$ clear of the surface of the donor eye when screwed down to its fullest extent.

By carefully screwing the main body clockwise on the pressure-adjusting ring the top clamp will be caused to lower on to the scleral surface of the donor eye, which will be engaged by the 'teeth' on the inner aspect of the clamp ring. The more the body is screwed clockwise, obviously the greater will be the compression applied to the globe (Fig. 2).

When a suitable tension for trephining or lamellar dissection is judged to have been reached, the

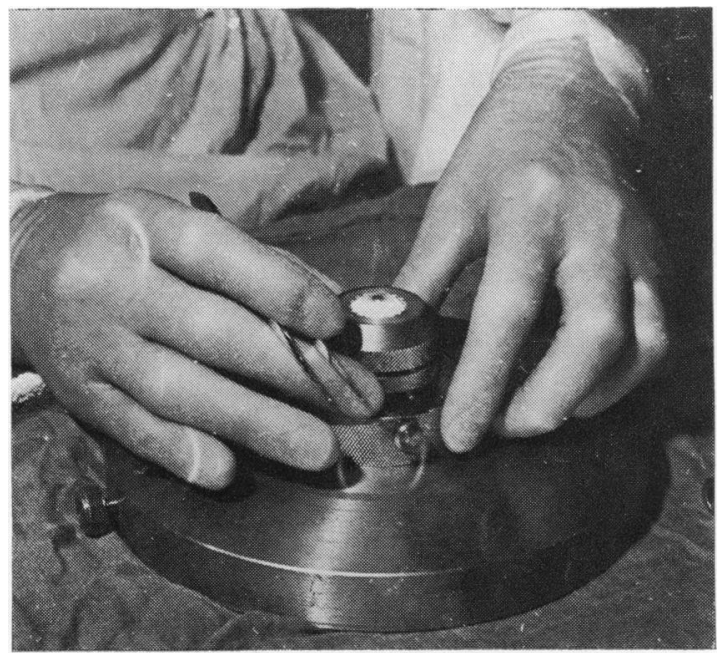

Fig. 2 Adjusting the pressure in the donor eye

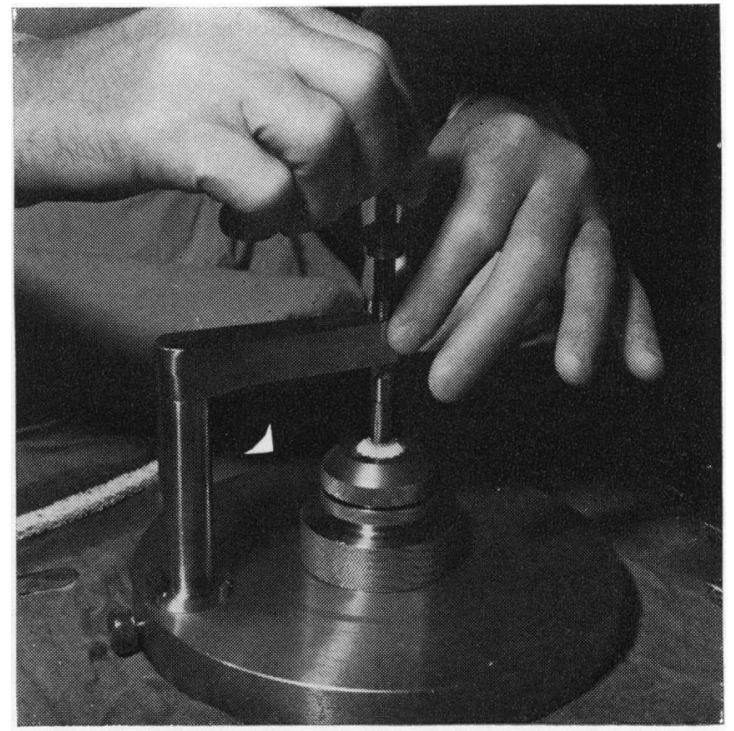

Fig. 3 Using the trephine

locking screw through the pressure adjuster is tightened so that the main body and the pressure adjuster will then rotate as a single unit.

A disposable trephine blade, having been selected, is fitted securely on to its appropriate adaptor, and screwed into the lower end of the trephine assembly.

The support arm is then slotted into position, and provided the donor cornea has been properly centred on the central axis of rotation the surgeon may proceed with an accurately centred and perfectly vertical trephination on an eye with a constant intraocular pressure (Fig. 3). After the anterior chamber has been entered, if the trephine is incomplete it may then be completed with some suitable sharp blade or scissors, the central spindle allowing easy rotation with fingertip control.

\section{Removal of a corneoscleral disc for storage or punching}

The donor eye is placed in the same assembly as above, except that one uses a top clamp with a wider inner aperture.

The eye pressure as above having been adjusted, rotating the eye on a sharp blade allows for simple and atraumatic removal of a corncal disc with a generous scleral rim.

\section{Punching a penetrating donor disc from the} endothelial surface

The corneoscleral disc is obtained as above. The top clamp is removed and the donor eye removed from the eye support. A Teflon plate with a concave 
upper surface is then placed inside the main body on top of the eye support.

The assembled trephine is placed through the aperture in the supporting arm, which is then swung into the central position and locked. The adjusting screw ' $\mathrm{C}$ ' on the trephine is adjusted so as to allow the trephine blade just to fail to meet the surface of the Teflon plate. The trephine is swung aside, and a thin disposable silicone pad placed in the concavity of the Teflon plate. The corneoscleral disc is then centred on the surface with the endothelial surface uppermost and the smaller top clamp is screwed down to its full extent.

By screwing the main body of the central assembly into the adjusting ring the teeth of the top clamp will fasten the corneoscleral disc securely to the Teflon plate and silicone pad. The trephine is then returned to and locked in the central position and the donor disc punched out. The trephine will pass through the cornea and into the soft thin pad of silicone rubber but will not be damaged by impinging on the Teflon plate, as screw ' $C$ ' will limit the travel of the body of the trephine through the supporting arm.

\section{Discussion}

Because the ideal way of cutting a donor disc is not satisfactorily defined and because donor material may take the form of either a whole eye or a prepared corneoscleral disc, we have devised an instrument which we believe brings to this surgical task an element of precision not previously obtainable. With practice and experience the small extra effort involved takes very little extra time, and we find the results are rewarding. 